\title{
Entanglement dynamics in dispersive optomechanics: Nonclassicality and revival
}

\author{
Igor Brandão $\odot,{ }^{*}$ Bruno Suassuna $\odot,{ }^{\dagger}$ Bruno Melo $\odot,{ }^{\ddagger}$ and Thiago Guerreiro ${ }^{\S}$ \\ Department of Physics, Pontifical Catholic University of Rio de Janeiro, Rio de Janeiro 22451-900, Brazil
}

(Received 5 June 2020; revised 22 October 2020; accepted 7 December 2020; published 28 December 2020)

\begin{abstract}
We study entanglement dynamics in a dispersive optomechanical system consisting of two optical modes and a mechanical oscillator inside an optical cavity. The two optical modes interact with the mechanical oscillator, but not directly with each other. The appearance of optical entanglement witnesses nonclassicality of the oscillator. We study the dependence of the entanglement dynamics on the optomechanical coupling, the mean photon number in the cavity, and the oscillator temperature. An experimental realization with ultracold atomic ensembles is proposed.
\end{abstract}

DOI: 10.1103/PhysRevResearch.2.043421

\section{INTRODUCTION}

Entanglement is one of the most striking phenomena of quantum theory [1]. Generating, manipulating, and measuring entanglement in systems with many constituents and with a large number of degrees of freedom is one of the challenges of quantum information and metrology [2] and an interesting frontier in fundamental physics [3]. In particular, entangling massive objects could open the way to interesting tests of quantum theory [4,5] and experiments aimed at probing gravitational effects of quantum mechanical matter [6-11]. Optomechanical systems provide a resourceful platform to this end.

It is well known that entanglement of massive objects can be realized in quantum cavity optomechanical experiments [12]. For instance, a cavity with a moving end mirror can be used to generate entangled "cat states" of both light $[13,14]$ and matter [15-18]. Similar systems have also been proposed as an effective nonlinear medium [19-21], and squeezing [22-24] and optical entanglement [25] have been experimentally demonstrated in a variety of setups such as cavity cold atomic ensembles [26], dispersive dielectric membranes [27], and silicon microresonators [28]. In the linearized regime, particularly, entanglement dynamics [29,30] and stationary entanglement [31-34] have attracted much attention as these systems have a wide applicability ranging from precision force measurements [34,35] to quantum networks [36,37].

Certifying quantumness of optomechanical systems, however, is a far-from-obvious task. Relations among entanglement and nonclassicality measures of quantum states can be

\footnotetext{
*igorbrandao@aluno.puc-rio.br

†bruno.b.suassuna@gmail.com

*brunomelo@aluno.puc-rio.br

§arbosa@puc-rio.br
}

Published by the American Physical Society under the terms of the Creative Commons Attribution 4.0 International license. Further distribution of this work must maintain attribution to the author(s) and the published article's title, journal citation, and DOI. used to probe the quantum nature of inaccessible objects such as a harmonic oscillator in an optical cavity [38]. Recurrence of optical squeezing in a cavity with a moving mirror has also been proposed as a witness of nonclassicality [39], and it has been shown that when two subsystems locally interact with a third one, but not directly with each other, the appearance of entanglement among those subsystems is sufficient to prove nonclassicality of the third party [40]. Building on some of these ideas the present work studies the entanglement dynamics of a dispersive optomechanical system, how to use that dynamics to probe the quantum nature of the oscillator through optical degrees of freedom, and how to optimize the generated optical entanglement by careful choice of the optomechanical coupling and the number of photons in an experiment.

Considering as possible implementations levitated optomechanical systems, such as silica nanospheres or cold atomic ensembles, and a "two-sided" cavity with a moving mirror in the middle, we map how entanglement appears and evolves among the various optical and mechanical subsystems for different optomechanical coupling strengths and optical field intensities. The appearance of mechanically induced optical entanglement and its subsequent death and revival are generic in these systems and thus could be used to probe the quantization of the center-of-mass of the moving object in real experiments. We also point out in a simplified context that under certain circumstances entanglement seems to "flow" through different subsystems, and such dynamics can be used to infer nonclassicality and entanglement among different components of the system. We consider examples of both non-Gaussian and Gaussian initial quantum states, for which we study the dynamics of concurrence and the Duan criteria [41], respectively. An experiment using levitated cold atomic ensembles is proposed.

\section{HAMILTONIAN DESCRIPTION}

The system we are primarily interested in is shown in Fig. 1(a): two optical cavities, of lengths $L_{a}$ and $L_{b}$, are populated by modes of frequencies $\omega_{a}$ and $\omega_{b}$ and share a common 
(a)

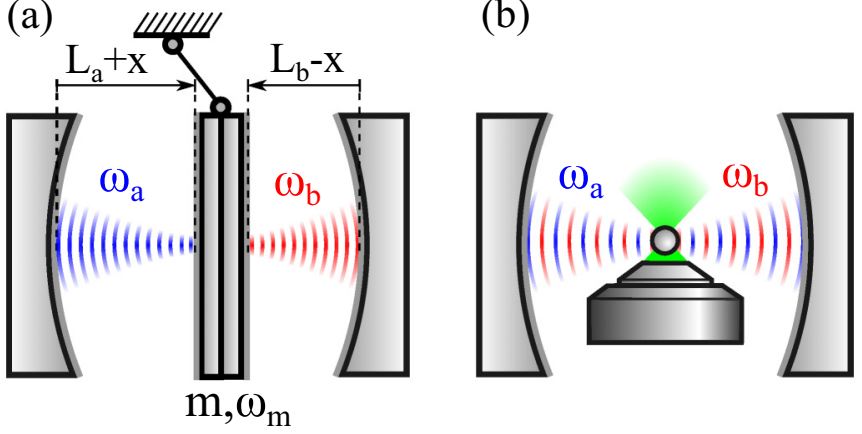

FIG. 1. (a) Schematics of coupled optical cavities sharing a "mirror-in-the-middle" under a harmonic potential. No photon transfer from one cavity to the other is allowed. (b) Schematics of a particle trapped by an optical tweezer coupled to two modes of a cavity. The particle can be considered as a silica nanosphere or a cloud of ultracold atoms. When the levitated object is properly positioned, the Hamiltonian describing both systems acquires the same form.

perfect movable mirror of mass $m$ subject to a harmonic potential of frequency $\omega_{m}$. We refer to this as the "mirror-inthe-middle" configuration. In this system the optical modes never interact directly, except via the dispersive coupling due to the presence of the mechanical mode. Since we are interested in studying entanglement dynamics in optomechanics, we assume the cavities can be initialized in particular states and the laser driving term can be turned off during the course of the experiment. It is also assumed that optical losses are negligible during the time of the experiment, and a discussion of the conditions under which this is true and the experimental feasibility are addressed in the experimental proposal.

The Hamiltonian of the system reads [12]

$$
\begin{aligned}
\frac{H}{\hbar}= & \omega_{a} \hat{a}^{\dagger} \hat{a}+\omega_{b} \hat{b}^{\dagger} \hat{b}+\omega_{m} \hat{c}^{\dagger} \hat{c} \\
& -g_{0, a} \hat{a}^{\dagger} \hat{a}\left(\hat{c}^{\dagger}+\hat{c}\right)+g_{0, b} \hat{b}^{\dagger} \hat{b}\left(\hat{c}^{\dagger}+\hat{c}\right),
\end{aligned}
$$

where $g_{0, i}=\omega_{i} x_{\mathrm{ZPF}} / L_{i}$ are the optomechanical couplings, with $x_{\mathrm{ZPF}}=\sqrt{\hbar / 2 m \omega_{m}}$ being the zero-point fluctuation of the mirror, and $\hat{a}, \hat{b}$, and $\hat{c}\left(\hat{a}^{\dagger}, \hat{b}^{\dagger}\right.$, and $\left.\hat{c}^{\dagger}\right)$ are the annihilation (creation) operators of each optical and mechanical modes, denoted by $A, B$, and $C$, respectively. Such a Hamiltonian can also be implemented using a cavity with a levitated nanoparticle [42-44] or an ultracold atom cloud [26,45,46] properly positioned along the cavity axis. This is illustrated in Fig. 1(b) (see Appendices A and B for details).

Assuming equal frequencies for the optical modes $\omega_{a}=$ $\omega_{b}$ and approximately equal cavity lengths $L_{a} \sim L_{b}$, we may simplify the notation and directly write $g_{0} \equiv g_{0, a} \sim g_{0, b}$. The unitary evolution operator resulting from Eq. (1) becomes

$$
\begin{aligned}
\hat{U}(t)= & e^{-i \hat{c}^{\dagger} \hat{c} t} e^{-i r_{a} \hat{a}^{\dagger} \hat{a} t} e^{-i r_{b} \hat{b}^{\dagger} \hat{b} t} \\
& \times e^{k\left(\hat{a}^{\dagger} \hat{a}-\hat{b}^{\dagger} \hat{b}\right)\left(\hat{\imath} \eta(t)-\hat{c}^{\dagger} \eta(t)^{*}\right)} e^{-i\left(\hat{a}^{\dagger} \hat{a}-\hat{b}^{\dagger} \hat{b}\right)^{2} B(t)},
\end{aligned}
$$

where we define the dimensionless optomechanical coupling $k=g_{0} / \omega_{m}$, the "normalized frequencies" $r_{i}=\omega_{i} / \omega_{m}$, the scaled time $\omega_{m} t \rightarrow t$, and the functions $\eta(t)=1-e^{-i t}$ and $B(t)=-k^{2}(t-\sin t)$. Note the evolution operator is com- posed of a Kerr-like term, responsible for an effective optical nonlinearity [47], as well as an optically driven displacement operator acting on the mechanical mode.

It is expected that a generic separable state will evolve into an entangled one by virtue of the unitary evolution (2). We note that if an initially separable state gives birth to optical entanglement then there will certainly be its entanglement death. This springs from the fact that when $B(t)=2 \pi n, n \in \mathbb{N}$, the term in the evolution operator responsible for entangling the optical modes reduces to the identity operator at those times, therefore preserving the separability of the initial state. Analogous arguments show that optomechanical entanglement must also face death when $\eta(t)=0$.

Not every state will evolve to an entangled one, as can be seen by considering the energy eigenstates of the system

$$
\hat{\mathcal{D}}_{C}\left[k\left(n_{A}-m_{B}\right)\right]\left|n_{A}, m_{B}, \ell_{C}\right\rangle,
$$

where $\left\{\left|n_{A}, m_{B}, \ell_{C}\right\rangle\right\}$ denotes the number basis and $\hat{\mathcal{D}}_{C}(\alpha)$ the displacement operator acting on the mechanical oscillator, mode $C$, by a displacement $\alpha \in \mathbb{C}$ (see Appendix $\mathrm{C}$ for details).

\section{QUBIT STATES}

Consider the cavities in Fig. 1(a) initially populated by the state

$$
|\Psi(0)\rangle=\left(\frac{|0\rangle+|1\rangle}{\sqrt{2}}\right) \otimes\left(\frac{|0\rangle+|1\rangle}{\sqrt{2}}\right) \otimes|0\rangle .
$$

We refer to these as "qubit states" as they are restricted to the vacuum-one-photon subspace. These states can be prepared in quantum optics in an approximate way using photon pair sources and displacement-based detection $[48,49]$ or nonlinear light-matter interactions in cavity quantum electrodynamics [50,51]. In levitated quantum electrodynamics [52], it is also possible to couple two qubits to a nanosphere or rotor according to the interaction Hamiltonian (1), with the qubits assuming the role of the optical fields and the levitated object the role of the mirror-in-the-middle. Among the advantages of this type of scheme is the fact that the optomechanical coupling admits a wide tunability, potentially allowing tests of the optomechanical interaction in novel regimes. Moreover, read-out of the "optical" modes can be achieved through standard qubit read-out techniques [53]. The mirror-in-the-middle is taken to be in the ground state for simplicity; in the next section we consider the effects of a finite-temperature oscillator. Notice the initial state is separable and hence the appearance of entanglement between modes $A$ and $B$ would evidence the nonclassical nature of mode $C$ [40]. Time evolution of (4) in the interaction picture is explicitly given by

$$
\begin{aligned}
|\Psi(t)\rangle= & \frac{|00\rangle}{2}|0\rangle+e^{i B(t)} \frac{|01\rangle}{2} \hat{\mathcal{D}}_{C}(k \xi(t))|0\rangle \\
& +e^{i B(t)} \frac{|10\rangle}{2} \hat{\mathcal{D}}_{C}(-k \xi(t))|0\rangle+\frac{|11\rangle}{2}|0\rangle,
\end{aligned}
$$

where $\xi(t)=e^{i t} \eta(t)$. The evolved state (5) exhibits entanglement between modes $A$ and $B$. This can be promptly seen by noticing that coherent states are nonorthogonal and, in the limit of small coupling $k$, the state assumes the form 
$|\Psi(t)\rangle \simeq\left|\varphi_{A B}\right\rangle \otimes\left|\varphi_{C}\right\rangle$, where

$$
\begin{aligned}
\left|\varphi_{A B}\right\rangle \simeq & \frac{|0\rangle}{2}\left(|0\rangle+e^{i B(t)}|1\rangle\right) \\
& +e^{i B(t)} \frac{|1\rangle}{2}\left(|0\rangle+e^{-i B(t)}|1\rangle\right)
\end{aligned}
$$

and $\left|\varphi_{C}\right\rangle \simeq|0\rangle$. For times $t$ such that $|B(t)| \simeq \pi / 2+2 \pi n$, $n \in \mathbb{N}$, the state $\left|\varphi_{A B}\right\rangle$ becomes maximally entangled. The origin of this entanglement can be heuristically explained by a simple argument: the ground state of the mechanical oscillator is a Gaussian wave packet in the position basis. Each possible position adds up coherently, introducing correlations in the lengths of the left and right cavities in Fig. 1(a). This imprints correlations in the phases of the corresponding electromagnetic fields in modes $A$ and $B$, giving rise to entanglement. As long as the dimensionless optomechanical coupling $k$ due to radiation pressure on the middle mirror is sufficiently small, mode $C$ will be approximately unperturbed and therefore, to a good approximation, disentangled from $A B$. On the other hand, as the coupling strength increases, mode $C$ can become significantly entangled with modes $A$ and $B$; the question of entanglement among different subsystems as a function of $k$ is addressed in the next section.

To quantitatively evaluate the entanglement in Eq. (5) we calculate the three-partite density matrix $\rho_{A B C}=$ $|\Psi(t)\rangle\langle\Psi(t)|$, from which we obtain the reduced state $\rho_{A B}=$ $\operatorname{Tr}_{C}\left(\rho_{A B C}\right)$ :

$$
\rho_{A B}(t)=\frac{1}{4}\left(\begin{array}{cccc}
1 & e^{C(t)} & e^{C(t)} & 1 \\
e^{C^{*}(t)} & 1 & e^{-2 k^{2}|\eta(t)|^{2}} & e^{C^{*}(t)} \\
e^{C^{*}(t)} & e^{-2 k^{2}|\eta(t)|^{2}} & 1 & e^{C^{*}(t)} \\
1 & e^{C(t)} & e^{C(t)} & 1
\end{array}\right),
$$

with $C(t)=i B(t)-k^{2}|\eta(t)|^{2} / 2$. Notice that for small values of $k$ the mirror is "weakly entangled" with modes $A$ and $B$ and some of the off-diagonal terms of the reduced density matrix acquire exponentials that alternate between periods of decay and periods of growth. This can be seen as an example of a weak form of decoherence and "non-Markovian" evolution for the partitions of the whole system, in which information about the optical modes leak into correlations with the mirror and is later retrieved. The mirror introduces a "memory" in the system [54]. Non-Markovianity springs from the fact that the mirror is part of the system under study and hence its degrees of freedom are under control.

Since the state $\rho_{A B}(t)$ is restricted to the subspace spanned by $\{|0\rangle,|1\rangle\}$, we can use concurrence as a measure of entanglement. As an example, plots of the concurrence $\mathcal{C}_{A B}(t)$ and the von Neumann entropy $S_{A B}(t)$ of $\rho_{A B}$ are shown in Fig. 2 for the coupling value $k=0.5$. The optical modes exhibit positive concurrence and hence entanglement as a function of time. Moreover, the system exhibits sudden death and birth of entanglement. It is also possible to see that the entropy, which is initially zero, oscillates as a function of time. This is another indication of the non-Markovian nature of the system. A nonzero entropy of $A B$ signals entanglement among the three-partite system $A B C$. Moreover, note that the maxima of concurrence (entanglement of $A B$ ) coincide with the minima of entropy (entanglement of $A B C$ ). This suggests that, after emerging in the system, entanglement "flows" (during the

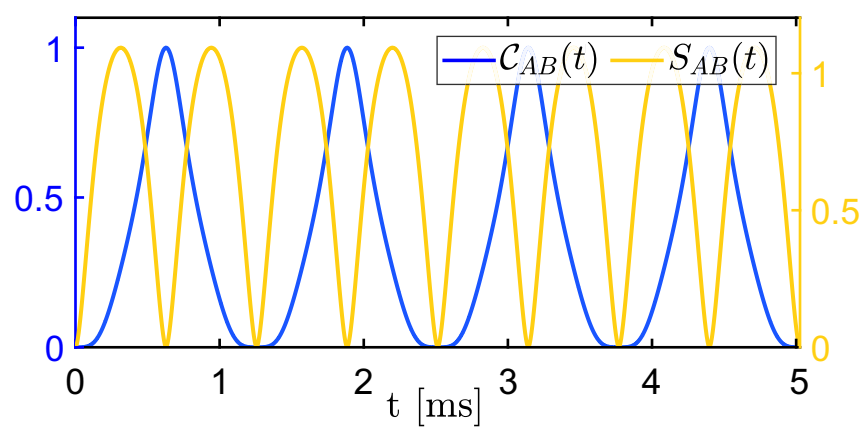

FIG. 2. Concurrence (blue line) and von Neumann entropy (yellow line) for $\rho_{A B}(t)$ as a function of time. For this plot, $k=0.5$.

limited period of its lifetime) among different partitions of the system.

\section{CONTINUOUS VARIABLE STATES AND FINITE TEMPERATURE}

We now consider a scenario in which initially the optical modes are populated by monochromatic coherent states and the moving object (sphere, cloud of atoms, or mirror) is in a thermal state at temperature $T$ :

$$
\rho(0)=|\alpha\rangle\langle\alpha|\otimes| \beta\rangle\left\langle\beta\left|\otimes \frac{1}{Z} \sum_{n} e^{-\frac{n h \omega_{m}}{k_{B} T}}\right| n\right\rangle\langle n|,
$$

where $Z=\sum_{n} e^{-\frac{n \hbar \omega_{m}}{k_{B} T}}$ is the thermal partition function. Note that although the initial state here considered is Gaussian, the Hamiltonian (1) has cubic terms in creation and annihilation operators and, therefore, does not preserve Gaussianity [55]. In order to study the dynamics of entanglement for continuous variable states, we turn our attention to the time-dependent Duan criteria [41].

We define $D_{i j}$ as an average of the Einstein-PodolskiRosen (EPR) variance for modes $i$ and $j$ :

$$
D_{i j} \equiv \frac{1}{2}\left[\left(\Delta \hat{u}_{i j}\right)^{2}+\left(\Delta \hat{v}_{i j}\right)^{2}\right]
$$

where

$$
\begin{aligned}
& \hat{u}_{i j}=\hat{x}_{i}+\hat{x}_{j}, \\
& \hat{v}_{i j}=\hat{p}_{i}-\hat{p}_{j}
\end{aligned}
$$

are the EPR operators for different modes, $i, j=A, B, C$, and $(\Delta \hat{o})^{2} \equiv\left\langle\hat{o}^{2}\right\rangle-\langle\hat{o}\rangle^{2}$ denotes the variance of an observable $\hat{o}$. The Duan criteria states that any separable state satisfies

$$
D_{i j} \geqslant 1 \text {. }
$$

Therefore, if at any time $t$ a violation of the above inequality is observed, modes $i$ and $j$ are necessarily entangled at that time. As the Duan criteria is written in terms of field quadratures, it can be promptly measured with homodyne detection techniques readily available in the laboratory [56]. We also note that when $t=2 \pi n / \omega_{m}, n \in \mathbb{N}$, the unitary evolution (2) acts analogous to a $\chi^{(3)}$ interaction in nonlinear optics [57]. In these moments, the optomechanical system behaves as a nonlinear optical source of squeezing, verified numerically, 


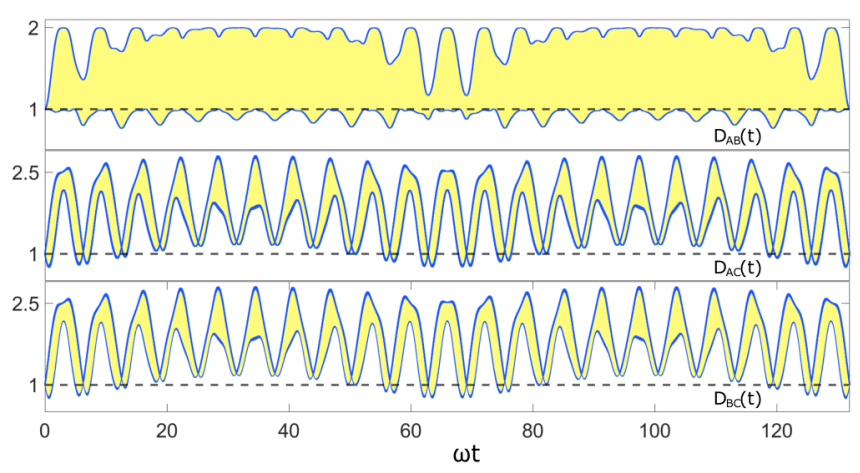

FIG. 3. Time-dependent EPR variances $D_{i j}(t)$ (yellow shading) for the various bipartitions of the system, with its envelope (blue line) and threshold for the Duan criteria (black dashed line). We use $\omega_{a}=\omega_{b} \simeq 2 \pi \cdot 1.59 \times 10^{14} \mathrm{~Hz}$ and the remaining parameters as in Table I.

from which quantum correlations can be read out from the leaking fields of the cavity [58].

Given the time evolution operator in Eq. (2) we are able to find analytical expressions for the EPR variance of every bipartition of the system (see Appendix D for details). For the optical modes we have

$$
\begin{aligned}
D_{A B}(t)= & 1+\left[|\alpha|^{2}+2 \alpha \beta \cos \left[\left(r_{a}+r_{b}\right) t\right]+|\beta|^{2}\right] \\
& -\left[|\alpha|^{2}+2 \alpha \beta \cos \left[\left(r_{a}+r_{b}\right) t+2 B(t)\right]+|\beta|^{2}\right] \\
& \times e^{-2\left[|\alpha|^{2}+|\beta|^{2}\right][1-\cos (2 B(t))]} e^{-k^{2}|\eta(t)|^{2}[2 \bar{n}+1]},
\end{aligned}
$$

where $\bar{n}$ is the thermal occupation number for the mechanical oscillator.

Figure 3 shows the time-evolving EPR variances for different bipartitions of the system: $A B$ (opto-opto), $B C$ (optomechanical), and $A C$ (optomechanical). We once again observe periodic birth of entanglement and, from the discussion in Sec. II, we can assert that there is death and revivals of entanglement for every bipartition of the system. Moreover, as in the qubit case, the appearance of entanglement between optical modes given the initially separable state can be used in experiments to probe the nonclassicality of the mechanical mode [40]. Although the analytical formulas for the EPR variance are rather involved, it is possible to obtain insight into the entanglement dynamics by looking into their periodicity.

For typical optical and mechanical frequencies, the term $\cos \left[\left(r_{a}+r_{b}\right) t\right]$ represents fast oscillations that do not contribute significantly to the overall envelope of the EPR variances. Consequently, when $k \ll 1 / \sqrt{2}$, Eq. (13) is dominated by the term $\exp \left\{-2\left[|\alpha|^{2}+|\beta|^{2}\right][1-\cos (2 B(t))]\right\}$, which has a period of $\tau=\pi / k^{2}$. We call this the "lowcoupling" regime. On the other hand, if $k \gg 1 / \sqrt{2}$ the variance is dominated by the term $\exp \left\{-k^{2}|\eta(t)|^{2}[2 \bar{n}+1]\right\}$, of period $\tau=2 \pi$. We refer to this as the "high-coupling" regime. The periodicity of these functions dictates the overall periodicity of the envelope of the EPR variances. Going back to nonscaled time we make the substitution $\tau \rightarrow \tau / \omega_{m}$. Then, for values in the low-coupling regime, observation of EPR variance revivals are only possible when $\pi / \omega_{m} k^{2} \ll \kappa^{-1}$, where $\kappa^{-1}$ is the inverse cavity linewidth, or the approximate photon lifetime in the cavity. This translates into the so-called
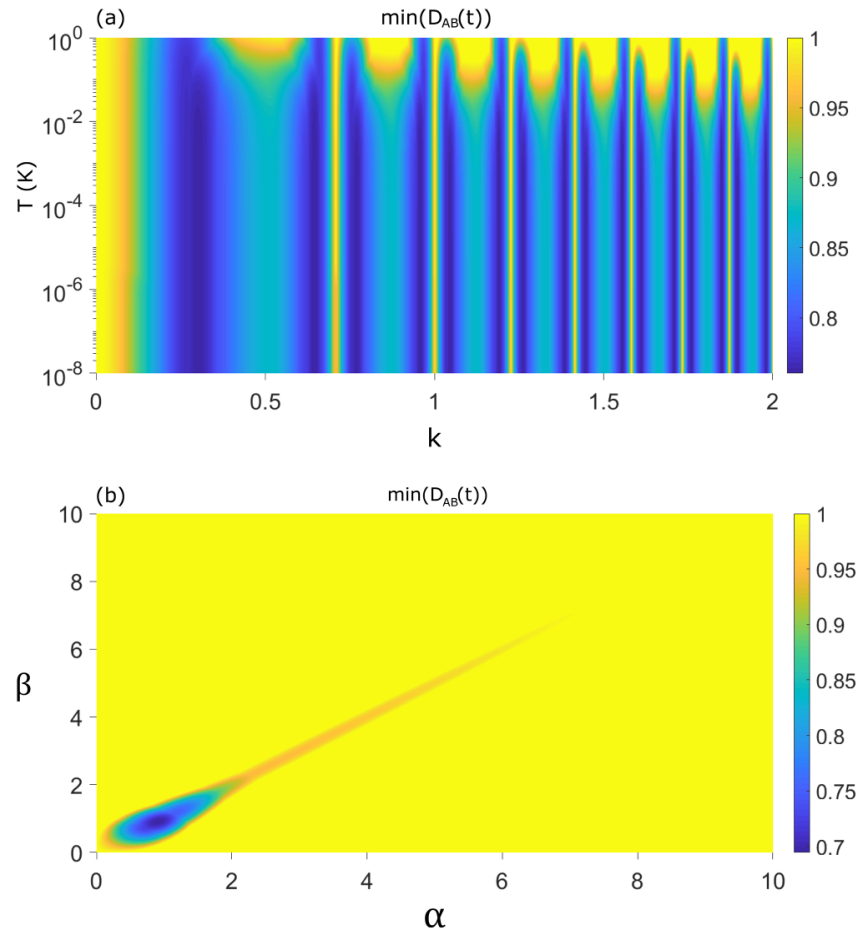

FIG. 4. (a) Minimum value of $D_{A B}(t)$ within the photon lifetime inside the cavity, $\tau=\kappa^{-1} \sim 15.6 \mu \mathrm{s}$, as a function of (a) dimensionless coupling $k$ and mechanical oscillator's temperature $T$ and (b) coherent state amplitudes of $\alpha$ and $\beta$. The optimal coherent amplitudes that globally minimize the Duan criteria are found to be $\alpha \sim \beta \sim 0.91$. We use $\omega_{a}=\omega_{b} \simeq 2 \pi \cdot 1.59 \times 10^{14} \mathrm{~Hz}$ and every other parameter as in Table I.

photon blockade condition $g_{0}^{2} /\left(\omega_{m} \kappa\right) \gg 1$ [45,59]. For the high-coupling regime, observation of full entanglement dynamics is conditioned on satisfying $2 \pi / \omega_{m} \ll \kappa^{-1}$, which translates into the resolved-sideband regime $\omega_{m} \gg \kappa[60]$.

Knowledge of the behavior of the EPR variance as a function of optomechanical coupling and temperature is useful when designing an experiment. We observe that the optical entanglement in modes $A B$ is strongly affected by the dimensionless coupling $k$ in a nontrivial way. Figure 4(a) shows the minimum of the EPR variance for the optical modes within the photon lifetime $\kappa^{-1}$ inside the cavity as a function of coupling $k$ and oscillator initial temperature $T$. In the low-coupling regime, where $k \ll 1 / \sqrt{2}$, above a threshold coupling of $k \approx 0.1, \min \left(D_{A B}(t)\right)$ generally falls below 1 and hence the system always exhibits entanglement. In contrast, the Duan criteria is very sensitive to $k$ in the high-coupling regime $k \gg 1 / \sqrt{2}$, presenting $\min \left(D_{A B}(t)\right) \geqslant$ 1 in the vicinity of $2 k^{2}=N$, where $N$ is a positive integer. Under this condition the criteria becomes greater than 1 and no entanglement can be certified regardless of the oscillator temperature. An immediate implication is that, as $k$ grows, the separation between inconclusive regions becomes smaller. At high-coupling values, small uncertainties in the coupling can then place the system in a parameter region in which the Duan criteria is inconclusive, presenting challenges to an experiment. Therefore, increasing coupling may not be the 
TABLE I. Proposed values for the experimental implementation with ultracold atoms.

\begin{tabular}{lcc}
\hline \hline Parameter & Units & Value \\
\hline Number of atoms $N$ & & $5.43 \times 10^{5}$ \\
Trap frequency $\omega_{m} / 2 \pi$ & $\mathrm{kHz}$ & 95.49 \\
Coupling $k=\frac{g_{0}}{\omega_{m}}$ & & 0.74 \\
Mechanical dissipation $\Gamma / 2 \pi$ & $\mathrm{kHz}$ & 1 \\
Cavity finesse $\mathcal{F}$ & & $3 \times 10^{6}$ \\
Cavity length $L$ & $\mu \mathrm{m}$ & 783 \\
Cavity linewidth $\kappa / 2 \pi$ & $\mathrm{kHz}$ & 10.19 \\
Temperature $T$ & $\mu \mathrm{K}$ & 0.8 \\
Mean photon number $|\alpha|^{2}=|\beta|^{2}$ & & 0.25 \\
\hline \hline
\end{tabular}

best strategy for an experiment aimed at verifying optical entanglement.

Moreover we observe that entanglement persists well above the microkelvin temperatures reported in current optomechanical experiments [26,61-63], consistent with the theoretical results for the linearized regime in Ref. [64]. Conversely, from our numerical calculations, we have observed that although the minimum value of $D_{A B}(t)$ remains well below 1, the time spent below this threshold becomes increasingly smaller as the temperature grows higher, making it effectively harder to experimentally verify entanglement with the Duan criteria at microkelvin temperatures.

The mean number of photons in the cavity also plays an important role in optical entanglement generation. Figure 4(b) shows a surface plot of $\min \left(D_{A B}(t)\right)$ within the photon lifetime as a function of the coherent state amplitudes $\alpha$ and $\beta$, taken to be real numbers for simplicity. The Duan criteria can only be conclusive when the energy is approximately evenly distributed among the two optical modes, which happens when $\alpha \sim \beta$. For the parameters used, the optimal coherent amplitudes that minimize $D_{A B}(t)$ are found to be $\alpha \sim \beta \sim$ 0.91 .

\section{EXPERIMENTAL PROPOSAL}

With increasing advances in the field of quantum cavity optomechanics [61-63], experiments in the high-coupling and long-coherence-time regimes are expected, although observing entanglement as described in the present work remains challenging. One notable exception and a promising candidate is optomechanics with ultracold atomic ensembles, where a coherent cloud of atoms is trapped within an optical cavity by a standing wave (trap beam) and the collective center-of-mass coordinate effectively behaves as a quantum mechanical oscillator which can be monitored by a second optical mode (probe beam). Couplings as high as $k \approx 10$ have been reported in such ultracold experiments [26], and the system allows wide tunability of the relevant parameters.

In order to observe entanglement dynamics, the lifetime of a photon inside the cavity $1 / \kappa$ needs to be longer than the time necessary for optical entanglement to reach its first local maximum. Table I presents values for the relevant experimental parameters adapted from Ref. [26], where the motion of an ultracold gas of ${ }^{87} \mathrm{Rb}$ was studied. With these values the resulting photon lifetime is $\tau_{p}=15.7 \times 10^{-6} \mathrm{~s}$ and within this lifetime the minimum value for the optical EPR variance is found to be $D_{A B}(t) \approx 0.8$. This suggests that in these systems the observation of mechanically induced optical entanglement can be within reach.

Once $D_{A B}(t)<1$, predicted by Eq. (2) and shown in Fig. 3, the optomechanical interaction can be switched off by acting on the trap beam and moving the oscillator with respect to the nodes of the probe beam (see Appendix B). Quantum correlations can then be read out from the leaking probe field of the cavity by using homodyne detection techniques [58,65].

Moreover, following Ref. [26], we estimate the maximal heating rate of a cloud of atoms due to backaction from each optical mode, $R_{\mathrm{c}}=N g_{0}^{2} /(4 \Gamma \kappa) R_{\mathrm{fs}}$, and due to spontaneous emission, $R_{\mathrm{fs}}=\hbar^{2} k_{p}^{2} g_{0}^{2} \bar{n}_{\mathrm{cav}} \Gamma /\left(m \Delta_{\mathrm{ca}}^{2}\right)$, where $k_{p} \simeq$ $2 \pi / 780 \mathrm{~nm}^{-1}$ is the probe wave vector, $m \simeq 1.44 \times 10^{-25} \mathrm{~kg}$ is the atomic mass of ${ }^{87} \mathrm{Rb}, \bar{n}_{\text {cav }}$ is the mean photon number for each optical mode, and $\Delta_{\text {ca }} /(2 \pi) \simeq 209.16 \mathrm{GHz}$ is the detuning between the frequencies for the optical modes and the atomic resonance frequency. Given the proposed parameters, heating is dominated by the backaction heating rate, $R_{\mathrm{fs}} \ll$ $R_{\mathrm{c}} \simeq 5.98 \times 10^{-32} \mathrm{~J} / \mathrm{s}$. We note that, when compared to the phonon energy, the maximal heating rate is much smaller than phonon and photon loss decoherence rates, $R_{c} /\left(\hbar \omega_{m}\right) \simeq$ $9.45 \times 10^{-4} \mathrm{~Hz} \ll \kappa, \Gamma$. Therefore, reheating of the mechanical oscillator should play a negligible role during the course of the experiment.

\section{CONCLUSION}

In this article we have studied the entanglement and entropy dynamics of a mirror-in-the-middle optomechanical system. Implementations using levitated particles have been briefly discussed. We have seen that an initially separable quantum state can evolve to an entangled one, exhibiting birth, death, and revivals of entanglement and entropy for qubit and continuous-variable states; moreover, the appearance of entanglement in this setting evidences the nonclassical nature of the mechanical oscillator. Therefore, optical entanglement will arise if and only if the mechanical oscillator is quantum mechanical.

The entanglement dynamics is strongly influenced by the system parameters, notably the dimensionless optomechanical coupling $k=g_{0} / \omega_{m}$ and the mean number of photons in the experiment. We have shown the existence of two distinct regimes depending on whether $k<1 / \sqrt{2}$ or $k \geqslant 1 / \sqrt{2}$. In addition, we have observed that optical entanglement is maximized when the energy is evenly distributed in the optical modes and that it persists at microkelvin temperatures of the mechanical mode. These are valuable information when designing an experiment. Optomechanics with ultracold atomic ensembles present an interesting candidate for implementing the studied entanglement dynamics. Although a promising candidate, the dispersive Hamiltonian is not the only available platform to untangle the dynamics of entanglement and information flow in optomechanical systems. Exploring alternatives such as coherent scattering [61,63,66-69] might prove to be a very fruitful approach to observe entanglement and nonclassicality in experimental optomechanical systems. 


\section{ACKNOWLEDGMENTS}

This work was financed in part by the Serrapilheira Institute (Grant No. Serra-1709-21072); by Coordenacão de Aperfeiçoamento de Pessoal de Nível Superior, Brasil (CAPES), Finance Code 001; and by Conselho Nacional de Desenvolvimento Científico e Tecnológico (CNPq). I.B. is thankful for support received from FAPERJ Scholarship No. E-26/200.270/2020. T.G. is thankful for support received from FAPERJ Scholarship No. E-26/202.830/2019.

\section{APPENDIX A: IMPLEMENTATION USING LEVITATED NANOPARTICLES}

Consider the system shown in Fig. 1(b): a nanoparticle of radius $r$, mass $m$, and refractive index $n_{p}$ is trapped in a harmonic trap of frequencies $\omega_{j=x, y, z}$ created by an optical tweezer inside a cavity populated by two optical modes of frequencies, $\omega_{a}$ and $\omega_{b}$, and annihilation/creation operators, $\hat{a} / \hat{a}^{\dagger}$ and $\hat{b} / \hat{b}^{\dagger}$, respectively. The presence of the particle causes a position-dependent shift in the cavity's resonance frequencies, so that the Hamiltonian of this system becomes [44]

$$
\begin{aligned}
\frac{\hat{H}}{\hbar}= & \omega_{a} \hat{a}^{\dagger} \hat{a}-U_{0, a} \sin ^{2}\left[k_{a}\left(x_{0}+x\right)\right] \hat{a}^{\dagger} \hat{a}+\omega_{b} \hat{b}^{\dagger} \hat{b} \\
& -U_{0, b} \sin ^{2}\left[k_{b}\left(x_{0}+x\right)\right] \hat{b}^{\dagger} \hat{b}+\sum_{j=x, y, z} \omega_{j} \hat{c}_{j}^{\dagger} \hat{c}_{j},
\end{aligned}
$$

where $x_{0}$ is the position of the center of the trap, $x$ is the particle's displacement, $U_{0, i}=\omega_{i} \alpha /\left(2 \epsilon_{0} V_{i}\right)$ is the frequency shift when the particle is at an intensity maximum of the cavity, $\alpha=4 \pi \epsilon_{0} r^{3}\left(n_{p}^{2}-1\right) /\left(n_{p}^{2}+2\right)$ is the polarizability of the particle, $V_{i}$ and $k_{i}$ are the volume and the wave number of mode $i$, respectively, and $\hat{c}_{j} / \hat{c}_{j}^{\dagger}$ is the phonon annihilation/creation operators along axis $j=x, y$, and $z$. We do not consider driving terms since we are interested in the dynamics of an isolated cavity without the influence of external systems such as a driving laser, as explained in the main text.

The interaction terms may yield linear couplings between the optical modes and the sphere if $x_{0}, k_{a}$, and $k_{b}$ are properly chosen. Expanding $\sin ^{2} k_{i}\left(x_{0}+x\right)$ around $x_{0}$ gives

$$
\begin{aligned}
\sin ^{2}\left[k_{i}\left(x_{0}+x\right)\right]= & \sin ^{2}\left(k_{i} x_{0}\right)+k_{i} \sin \left(2 k_{i} x_{0}\right) x \\
& +2 k_{i}^{2} \cos \left(2 k_{i} x_{0}\right) x^{2}+\mathcal{O}\left(x^{3}\right) .
\end{aligned}
$$

Now, consider the particular case in which the frequencies of the optical modes are two consecutive resonance frequencies, and let $L=2 n\left(\lambda_{a} / 2\right)=(2 n+1)\left(\lambda_{b} / 2\right)$ without loss of generality. Then, if the sphere is placed near the center of the cavity at $x_{0}=L / 2+\lambda_{a} / 8 \approx L / 2+\lambda_{b} / 8$, we have

$$
\sin ^{2} k_{a}\left(x_{0}+x\right) \approx 1 / 2+k_{a} x
$$

and

$$
\sin ^{2} k_{b}\left(x_{0}+x\right) \approx 1 / 2-k_{b} x .
$$

Substituting these approximations in Eq. (A1) and disregarding the motion along the $y$ and $z$ axes, we get

$$
\begin{aligned}
\frac{\hat{H}}{\hbar}= & \omega_{a}^{\prime} \hat{a}^{\dagger} \hat{a}-g_{0} \hat{a}^{\dagger} \hat{a}\left(\hat{c}_{x}^{\dagger}+\hat{c}_{x}\right)+\omega_{b}^{\prime} \hat{b}^{\dagger} \hat{b} \\
& +g_{0} \hat{b}^{\dagger} \hat{b}\left(\hat{c}_{x}^{\dagger}+\hat{c}_{x}\right)+\omega_{x} \hat{c}_{x}^{\dagger} \hat{c}_{x},
\end{aligned}
$$

TABLE II. Values reported in Ref. [26].

\begin{tabular}{lcc}
\hline \hline Parameter & Units & Value \\
\hline $\begin{array}{l}\text { Number of atoms } \\
\text { Trap frequency } \omega_{m}\end{array}$ & $\mathrm{kHz}$ & $10^{5}$ \\
Coupling $k=\frac{g_{0}}{\omega_{m}}$ & & $2 \pi \times 40$ \\
Cavity finesse $\mathcal{F}$ & & 9.50 \\
Cavity length $L$ & $\mu \mathrm{m}$ & $5.8 \times 10^{5}$ \\
Cavity mirror's radius $R$ & $\mathrm{~cm}$ & 194 \\
Cavity linewidth $\kappa$ & $\mathrm{MHz}$ & 5 \\
Temperature $T$ & $\mu \mathrm{K}$ & $2 \pi \times 0.66$ \\
\hline \hline
\end{tabular}

where $\omega_{i}^{\prime}=\omega_{i}-U_{0, i} / 2$ and $g_{0}=U_{0, a} k_{a} x_{\mathrm{ZPF}} \approx U_{0, b} k_{b} x_{\mathrm{ZPF}}$ is the intended linear coupling, with $x_{\mathrm{ZPF}}=\sqrt{\hbar / 2 m \omega_{x}}$ being the zero-point fluctuation. This Hamiltonian is formally equivalent to that of a double-sided cavity with a moving mirror-in-the-middle analyzed in the main text, with the condition $g_{0, a} \approx g_{0, b}$ being met due to the use of consecutive resonance frequencies. As a final remark, note that the modes $A$ and $B$ are not used for cooling of the particle, which is necessary for observing optical entanglement in the system [40]. Cooling of the center-of-mass motion of the particle can be addressed in a number of ways, such as a feedback acting on the trapping laser [70,71], dispersive coupling with a third optical mode [60], or coherent scattering between the trapping beam and the optical cavity $[61,63,66]$.

\section{APPENDIX B: IMPLEMENTATION USING ULTRACOLD ATOMIC ENSEMBLES}

In ultracold atom optomechanical experiments, an atomic ensemble is trapped inside an optical cavity. Collective centerof-mass motion of the atoms alters the cavity resonance frequency. It is similar to the dispersive optomechanical experiments with levitated spheres described in the previous section, with the cloud of atoms playing the role of the levitated nanoparticle.

The optomechanical coupling between an ultracold atomic cloud and a cavity optical mode with wavelength $\lambda_{a}$ is [26]

$$
g_{0, a}=k_{a} N \frac{\alpha_{0}^{2}}{\Delta_{\mathrm{ca}}} \sin \left(2 k_{a} z_{0}\right) \sqrt{\frac{\hbar}{2 N m \omega_{m}}},
$$

where $k_{a}$ is the wave number, $N$ is the number of atoms, $\Delta_{\text {ca }}$ is the atom-cavity detuning, $m$ is the mass of a single atom, $\omega_{m}$ is the mechanical frequency, and $\alpha_{0}=\sqrt{d^{2} \omega_{c} /\left(2 \hbar \epsilon_{0} V_{c}\right)}$ is the atom-single photon coupling rate, with $d$ being the dipole moment for the transition between the relevant atomic levels and $V_{c}$ being the cavity mode volume. Reported values from Ref. [26] for these quantities are shown in Table II. As in the previous case of a levitated nanoparticle, we chose $\sin \left(2 k_{a} z_{0}\right)=1$. The wavelength $\lambda_{b}$ should be chosen so that $\sin \left(2 k_{b} z_{0}\right)=-1$, so as to provide $g_{0, a}=g_{0, b}$.

As discussed in the main text, for the entanglement dynamics experiment to be feasible, the photon lifetime $\tau_{p}$ must be greater than the entanglement period $\tau_{e}$. One of the main constraints in fulfilling this condition is set by the finesse of the cavity. Therefore, we look for the minimum value of $\tau_{e} / \tau_{p}$ by varying the cavity length $L$, the number of atoms $N$, and 
TABLE III. Optimal parameters for different cavity mirrors' radii.

\begin{tabular}{lcccc}
\hline \hline$R(\mathrm{~cm})$ & $L(\mu \mathrm{m})$ & $N\left(10^{5}\right)$ & $\omega_{m}(\mathrm{kHz})$ & $\mathcal{F}\left(10^{6}\right)$ \\
\hline 1 & 1211 & 3.85 & $2 \pi \times 95$ & 1.30 \\
2.5 & 1035 & 5.64 & $2 \pi \times 92$ & 1.57 \\
5 & 783 & 5.43 & $2 \pi \times 95$ & 2.01 \\
10 & 669 & 5.80 & $2 \pi \times 91$ & 2.47 \\
\hline \hline
\end{tabular}

the mechanical frequency $\omega_{m}$ around the values in Table II, and then we calculate the minimum finesse necessary to make $\tau_{e} / \tau_{p}<1$ given the optimal values of $L, N$, and $\omega_{m}$.

In doing these calculations, it is necessary to account for the changes in the mode volume, given by $V_{c}=\pi w_{a}^{2} L$, where

$$
w_{a}=\sqrt{\frac{\lambda_{a}}{2 \pi} \sqrt{L(2 R-L)}}
$$

is the mode's waist, and the changes in the cavity linewidth

$$
\kappa=\frac{\mathcal{F}}{v_{\mathrm{FSR}}},
$$

with $v_{\mathrm{FSR}}=c / 2 L$ being the cavity's free spectral range. Finally, it is important to make sure that $k=g_{0} / \omega_{m} \neq \sqrt{n / 2}$, where $n$ a positive integer, when the Duan criteria is inconclusive. We find that for $L=783 \mu \mathrm{m}, N=5.43 \times 10^{5}$, and $\omega_{m}=2 \pi \times 95 \mathrm{kHz} \approx 2 \pi \times 95.49 \mathrm{kHz}$, the dimensionless optomechanical coupling is $k=0.743$ and the entanglement period to photon lifetime ratio is $\tau_{e} / \tau_{p}=3.46$. The finesse should then be increased to $2.01 \times 10^{6}$, so that $\tau_{e} / \tau_{p} \simeq 1$ and entanglement becomes measurable. In the main text, the proposed finesse is about 1.5 times larger, so that $\tau_{e} / \tau_{p}=0.669$.

Another parameter that could be varied is the radii of the cavity mirrors. Considering $1,2.5,5$, and $10 \mathrm{~cm}$ as possible radii, we find the values presented in Table III. As we can see, the finesse constraint can be relaxed provided that a smaller radius is used. Overall, the necessary values for $N, \omega_{m}$, and $\mathcal{F}$ differ by less than 1 order of magnitude from reported values in the literature.

\section{APPENDIX C: THE UNITARY EVOLUTION}

The mirror-in-the-middle Hamiltonian can be written as

$$
\frac{\hat{H}}{\hbar}=\omega_{m} \hat{c}^{\dagger} \hat{c}+\omega_{a} \hat{a}^{\dagger} \hat{a}+\omega_{b} \hat{b}^{\dagger} \hat{b}-g_{0}\left(\hat{a}^{\dagger} \hat{a}-\hat{b}^{\dagger} \hat{b}\right)\left(\hat{c}+\hat{c}^{\dagger}\right),
$$

where $\hat{a}, \hat{b}$, and $\hat{c}$ denote annihilation operators for the optical mode in the left cavity $(A)$, the right cavity $(B)$, and the mechanical oscillator $(C)$, respectively. In what follows we work with the rescaled time $\omega_{m} t$, denoted henceforth by $t$, and introduce the dimensionless variables $r_{a}=\omega_{a} / \omega_{m}, r_{b}=\omega_{b} / \omega_{m}$, and $k=g_{0} / \omega_{m}$.

Define the unitary operator [15]

$$
\hat{E}(k)=\exp \left[k\left(\hat{a}^{\dagger} \hat{a}-\hat{b}^{\dagger} \hat{b}\right)\left(\hat{c}^{\dagger}-\hat{c}\right)\right] .
$$

The operator $\hat{E}(k)$ commutes with $\hat{a}^{\dagger} \hat{a}$ and $\hat{b}^{\dagger} \hat{b}$, but not with $\hat{c}$,

$$
\hat{E}(k)^{\dagger} \hat{c} \hat{E}(k)=\hat{c}+k\left(\hat{a}^{\dagger} \hat{a}-\hat{b}^{\dagger} \hat{b}\right)
$$

which is calculated through the general identity

$$
e^{-\hat{A}} \hat{B} e^{+\hat{A}}=\hat{B}+[\hat{B}, \hat{A}]+\frac{1}{2 !}[[\hat{B}, \hat{A}], \hat{A}]+\cdots
$$

Using Eq. (C3) and its adjoint, we have

$$
\hat{E}(k)^{\dagger} \frac{\hat{H}}{\hbar \omega_{m}} \hat{E}(k)=\hat{c}^{\dagger} \hat{c}+r_{a} \hat{a}^{\dagger} \hat{a}+r_{b} \hat{b}^{\dagger} \hat{b}-k^{2}\left(\hat{a}^{\dagger} \hat{a}-\hat{b}^{\dagger} \hat{b}\right)^{2} .
$$

Considering the number basis $\{|n, m, \ell\rangle\}$, we see by the equation above that $\hat{E}(k)|n, m, \ell\rangle$ are the energy eigenstates of the system. Those are of the form

$$
\hat{E}(k)|n, m, \ell\rangle=\hat{\mathcal{D}}_{C}[k(n-m)]|n, m, \ell\rangle,
$$

where we denote the displacement operator of the mechanical oscillator by $\hat{\mathcal{D}}_{C}(\kappa)=\exp \left(\kappa \hat{c}^{\dagger}-\kappa^{*} \hat{c}\right)$, where $\kappa$ is a complex number. The energies corresponding to the eigenstates above are

$$
E_{n, m, \ell}=\hbar \omega_{m} \ell+\hbar \omega_{a} n+\hbar \omega_{b} m-\hbar \omega_{m} k^{2}(n-m)^{2} .
$$

By exponentiation of Eq. (C5), the unitary evolution operator is found to be

$$
\hat{U}(t)=\hat{E}(k) e^{-i\left[\hat{c}^{\dagger} \hat{c}+r_{a} \hat{a}^{\dagger} \hat{a}+r_{b} \hat{b}^{\dagger} \hat{b}-k^{2}\left(\hat{a}^{\dagger} \hat{a}-\hat{b}^{\dagger} \hat{b}\right)^{2}\right] t} \hat{E}(k)^{\dagger} .
$$

Next, we recall that $e^{i \hat{c}^{\dagger} \hat{c} t} \hat{c}^{\dagger} e^{-i \hat{c}^{\dagger} \hat{c} t}=e^{i t} \hat{c}^{\dagger}$ and $e^{i \hat{c}^{\dagger} \hat{c} t} \hat{c} e^{-i \hat{c}^{\dagger} \hat{c} t}=$ $e^{-i t} \hat{c}$, from which we derive the following identity:

$$
\begin{aligned}
& e^{i \hat{c}^{\dagger} \hat{c} t} \hat{A}\left(\hat{c}^{\dagger}-\hat{c}\right) e^{-i \hat{c}^{\dagger} \hat{c} t}=\hat{A}\left(e^{i t} \hat{c}^{\dagger}-e^{-i t} \hat{c}\right) \\
& \Rightarrow e^{i \hat{c}^{\dagger} \hat{c} t} e^{\hat{A}\left(\hat{c}^{\dagger}-\hat{c}\right)} e^{-i \hat{c}^{\dagger} \hat{c} t}=e^{\hat{A}\left(e^{i t} \hat{c}^{\dagger}-e^{-i t} \hat{c}\right)}
\end{aligned}
$$

where $\hat{A}$ is any operator that commutes with both $\hat{c}$ and $\hat{c}^{\dagger}$.

Letting $\hat{A}=k\left(\hat{a}^{\dagger} \hat{a}-\hat{b}^{\dagger} \hat{b}\right)$ in the identity above, we conclude that

$$
\begin{aligned}
\hat{E}(k) e^{-i \hat{c}^{\dagger} \hat{c} t} & =e^{-i \hat{c}^{\dagger} \hat{c} t} e^{i \hat{c}^{\dagger} \hat{c} t} \hat{E}(k) e^{-i \hat{c}^{\dagger} \hat{c} t} \\
& =e^{-i \hat{c}^{\dagger} \hat{c} t} \exp \left[k\left(\hat{a}^{\dagger} \hat{a}-\hat{b}^{\dagger} \hat{b}\right)\left(\hat{c}^{\dagger} e^{i t}-\hat{c} e^{-i t}\right)\right],
\end{aligned}
$$

hence

$$
\begin{aligned}
\hat{E}(k) e^{-i \hat{c}^{\dagger} \hat{c} t} \hat{E}(k)^{\dagger} \\
=e^{-i \hat{c}^{\dagger} \hat{c} t} \times \exp \left[k\left(\hat{a}^{\dagger} \hat{a}-\hat{b}^{\dagger} \hat{b}\right)\left(\hat{c}^{\dagger} e^{i t}-\hat{c} e^{-i t}\right)\right] \\
\quad \times \exp \left[-k\left(\hat{a}^{\dagger} \hat{a}-\hat{b}^{\dagger} \hat{b}\right)\left(\hat{c}^{\dagger}-\hat{c}\right)\right] .
\end{aligned}
$$

From the commutator

$$
\frac{1}{2}\left[\hat{c}^{\dagger} e^{i t}-\hat{c} e^{-i t}, \hat{c}^{\dagger}-\hat{c}\right]=i \sin (t)
$$

it becomes straightforward to compute

$$
\begin{aligned}
& {\left[k\left(\hat{a}^{\dagger} \hat{a}-\hat{b}^{\dagger} \hat{b}\right)\left(\hat{c}^{\dagger} e^{i t}-\hat{c} e^{-i t}\right), k\left(\hat{a}^{\dagger} \hat{a}-\hat{b}^{\dagger} \hat{b}\right)\left(\hat{c}^{\dagger}-\hat{c}\right)\right]} \\
& \quad=2 i k^{2}\left(\hat{a}^{\dagger} \hat{a}-\hat{b}^{\dagger} \hat{b}\right)^{2} \sin (t) .
\end{aligned}
$$

Since this commutes with both $\left(\hat{a}^{\dagger} \hat{a}-\hat{b}^{\dagger} \hat{b}\right)\left(\hat{c}^{\dagger} e^{i t}-\hat{c} e^{-i t}\right)$ and $\left(\hat{a}^{\dagger} \hat{a}-\hat{b}^{\dagger} \hat{b}\right)\left(\hat{c}^{\dagger}-\hat{c}\right)$, Eq. (C11) can be simplified using the particular form of the Baker-Campbell-Haursdoff formula,

$$
e^{\hat{A}} e^{\hat{B}}=e^{\hat{A}+\hat{B}+\frac{1}{2}[\hat{A}, \hat{B}]},
$$


valid when operators $\hat{A}$ and $\hat{B}$ commute with $[\hat{A}, \hat{B}]$. Thus, the expression,

$$
\begin{aligned}
\hat{U}(t)= & e^{-i \hat{c}^{\dagger} \hat{c} t} e^{-i r_{a} \hat{a}^{\dagger} \hat{a} t} e^{-i r_{b} \hat{b}^{\dagger} \hat{b} t} \\
& \times e^{k\left(\hat{a}^{\dagger} \hat{a}-\hat{b}^{\dagger} \hat{b}\right)\left[\hat{c} \eta(t)-\hat{c}^{\dagger} \eta(t)^{*}\right]} e^{-i\left(\hat{a}^{\dagger} \hat{a}-\hat{b}^{\dagger} \hat{b}\right)^{2} B(t)}
\end{aligned}
$$

holds for the unitary evolution operator $\hat{U}(t)$, where $\eta(t)=$ $1-e^{-i t}$ and $B(t)=-k^{2}[t-\sin (t)]$. It is often convenient to work in the interaction picture, where we evolve states by the unitary operator

$$
\hat{U}_{\text {I.P. }}(t)=e^{k\left(\hat{a}^{\dagger} \hat{a}-b^{\dagger} \hat{b}\right)\left[\hat{c} \eta(t)-\hat{c}^{\dagger} \eta(t)^{*}\right]} e^{-i\left(\hat{a}^{\dagger} \hat{a}-\hat{b}^{\dagger} \hat{b}\right)^{2} B(t)} .
$$

The Heisenberg evolution of the annihilation operators $\hat{a}$, $\hat{b}$, and $\hat{c}$ are then derived as follows:

$$
\begin{aligned}
\hat{a}(t) & =U^{\dagger}(t) \hat{a} \hat{U}(t) \\
& =e^{-i\left[r_{a} t+B(t)\right]} e^{2 i B(t) \hat{b}^{\dagger} \hat{b}} e^{-2 i B(t) \hat{a}^{\dagger} \hat{a}} \hat{\mathcal{D}}_{C}(+k \xi(t)) \hat{a}, \\
\hat{b}(t) & =U^{\dagger}(t) \hat{b} \hat{U}(t) \\
& =e^{-i\left[r_{b} t+B(t)\right]} e^{-2 i B(t) \hat{b}^{\dagger} \hat{b}} e^{2 i B(t) \hat{a}^{\dagger} \hat{a}} \hat{\mathcal{D}}_{C}(-k \xi(t)) \hat{b}, \\
\hat{c}(t) & =U^{\dagger}(t) \hat{c} \hat{U}(t)=\hat{c} e^{-i t}+k\left(\hat{a}^{\dagger} \hat{a}-\hat{b}^{\dagger} \hat{b}\right) \eta(t),
\end{aligned}
$$

where $\xi(t)=e^{i t} \eta(t)=e^{i t}-1=-\eta(t)^{*}$. In deriving these expressions we made use of the relations

$$
\begin{gathered}
e^{i \hat{A} \hat{a}^{\dagger} \hat{a}} \hat{a} e^{-i \hat{A}^{\dagger} \hat{a}}=e^{-i \hat{A}} \hat{a}, \\
e^{i \hat{A}\left(\hat{a}^{\dagger} \hat{a}\right)^{2}} \hat{a} e^{-i \hat{A}\left(\hat{a}^{\dagger} \hat{a}\right)^{2}}=e^{-i \hat{A}\left(2 \hat{a}^{\dagger} \hat{a}+I\right)} \hat{a},
\end{gathered}
$$

applicable whenever $\hat{A}$ commutes with both $\hat{a}$ and $\hat{a}^{\dagger}$. For instance, Eq. (C19) is derived as

$$
\begin{aligned}
U^{\dagger}(t) \hat{c} \hat{U}(t)= & e^{-k\left(\hat{a}^{\dagger} \hat{a}-\hat{b}^{\dagger} \hat{b}\right)\left[\hat{c} \eta(t)-\hat{c}^{\dagger} \eta(t)^{*}\right]} \\
& \times e^{+i \hat{c}^{\dagger} \hat{c} t} \hat{c} e^{-i \hat{c}^{\dagger} \hat{c} t} e^{k\left(\hat{a}^{\dagger} \hat{a}-\hat{b}^{\dagger} \hat{b}\right)\left[\hat{c} \eta(t)-\hat{c}^{\dagger} \eta(t)^{*}\right]} \\
= & e^{-i t} e^{-k\left(\hat{a}^{\dagger} \hat{a}-\hat{b}^{\dagger} \hat{b}\right)\left[\hat{c} \eta(t)-\hat{c}^{\dagger} \eta(t)^{*}\right]} \\
& \times \hat{c} e^{k\left(\hat{a}^{\dagger} \hat{a}-\hat{b}^{\dagger} \hat{b}\right)\left[\hat{c} \eta(t)-\hat{c}^{\dagger} \eta(t)^{*}\right]} \\
= & \hat{c} e^{-i t}-k \eta(t)^{*} e^{-i t}\left(\hat{a}^{\dagger} \hat{a}-\hat{b}^{\dagger} \hat{b}\right)
\end{aligned}
$$

which leads to the desired result after noting that $-\eta(t)^{*} e^{-i t}=$ $\eta(t)$.

\section{APPENDIX D: DUAN CRITERIA}

A general sufficient condition for nonseparability of a bipartite continuous-variable system is provided by the Duan criteria [41]. For any bipartite quantum system with observables $\hat{X}_{1}, \hat{P}_{1}, \hat{X}_{2}$, and $\hat{P}_{2}$ satisfying the canonical commutation relations

$$
\left[\hat{X}_{j}, \hat{P}_{k}\right]=i \delta_{j k},
$$

separability of the state $\rho$ implies that

$$
D=\frac{\left[\Delta\left(\hat{X}_{1}+\hat{X}_{2}\right)\right]^{2}}{2}+\frac{\left[\Delta\left(\hat{P}_{1}-\hat{P}_{2}\right)\right]^{2}}{2} \geqslant 1 .
$$

In other words, if we verify that $D<1$, then we may conclude that the modes are in an entangled state. We stress that $D \geqslant 1$ provides no information: this inequality does not imply that the state is separable.

If the operators above are expressed in terms of annihilation and creation operators, defined by

$$
\hat{X}_{j}=\frac{1}{\sqrt{2}}\left(\hat{a}_{j}^{\dagger}+\hat{a}_{j}\right), \quad \hat{P}_{j}=\frac{i}{\sqrt{2}}\left(\hat{a}_{j}^{\dagger}-\hat{a}_{j}\right),
$$

then the following expression is useful:

$$
\begin{aligned}
D= & \left\langle\hat{a}_{1}^{\dagger} \hat{a}_{1}\right\rangle+\left\langle\hat{a}_{2}^{\dagger} \hat{a}_{2}\right\rangle+\left\langle\hat{a}_{1}^{\dagger} \hat{a}_{2}^{\dagger}\right\rangle+\left\langle\hat{a}_{1} \hat{a}_{2}\right\rangle \\
& -\left(\left\langle\hat{a}_{1}^{\dagger}\right\rangle+\left\langle\hat{a}_{2}\right\rangle\right)\left(\left\langle\hat{a}_{2}^{\dagger}\right\rangle+\left\langle\hat{a}_{1}\right\rangle\right)+1 .
\end{aligned}
$$

By simple algebraic manipulations, we find

$$
\begin{aligned}
D= & \left\langle\hat{a}_{1}^{\dagger} \hat{a}_{1}\right\rangle+\left\langle\hat{a}_{2}^{\dagger} \hat{a}_{2}\right\rangle+2 \operatorname{Re}\left(\left\langle\hat{a}_{1} \hat{a}_{2}\right\rangle-\left\langle\hat{a}_{1}\right\rangle\left\langle\hat{a}_{2}\right\rangle\right) \\
& -\left|\left\langle\hat{a}_{1}\right\rangle\right|^{2}-\left|\left\langle\hat{a}_{2}\right\rangle\right|^{2}+1 .
\end{aligned}
$$

For the optical modes, $\hat{a}_{1} \rightarrow \hat{a}$ and $\hat{a}_{2} \rightarrow \hat{b}$, this gives

$$
\begin{aligned}
D_{A B}(t)= & 1+\left[|\alpha|^{2}+2 \alpha \beta \cos \left[\left(r_{a}+r_{b}\right) t\right]+|\beta|^{2}\right] \\
& -\left[|\alpha|^{2}+2 \alpha \beta \cos \left[\left(r_{a}+r_{b}\right) t+2 B(t)\right]+|\beta|^{2}\right] \\
& \times e^{-2\left[|\alpha|^{2}+|\beta|^{2}\right][1-\cos (2 B(t))]} e^{-k^{2}|\eta(t)|^{2}[2 \bar{n}+1]}, \quad \text { (D6) }
\end{aligned}
$$

assuming $\alpha, \beta \in \mathbb{R}$ for simplicity. The corresponding quantity between an optical mode and a mechanical mode, say $\hat{a}_{1} \rightarrow \hat{a}$ and $\hat{a_{2}} \rightarrow \hat{c}$, is given by

$$
\begin{aligned}
D_{A C}(t)= & 1+|\alpha|^{2}+\bar{n}+2 \operatorname{Re}(R(\alpha, t)) \\
& +|\eta(t)|^{2}\left[k^{2}\left[|\alpha|^{2}+|\beta|^{2}+\left(|\alpha|^{2}-|\beta|^{2}\right)^{2}\right]\right. \\
& \left.-k\left[|\alpha|^{2}-|\beta|^{2}\right]\right] \\
& -|\alpha|^{2} e^{-2\left[|\alpha|^{2}+|\beta|^{2}\right][1-\cos (2 B(t))]} e^{-k^{2}|\eta(t)|^{2}[2 \bar{n}+1],}
\end{aligned}
$$

where

$$
\begin{aligned}
R(\alpha, t) \equiv & \alpha e^{-|\alpha|^{2}\left(1-e^{-2 i B(t)}\right)-|\beta|^{2}\left(1-e^{2 i B(t)}\right)} \\
& \times\left[\bar{n}+1-|\alpha|^{2}\left(1-e^{-2 i B(t)}\right)+|\beta|^{2}\left(1-e^{2 i B(t)}\right)\right] \\
& \times k \eta(t) e^{-i\left[r_{a} t+B(t)\right]} e^{-k^{2}|\eta(t)|^{2} / 2} e^{-\bar{n} k^{2}|\eta(t)|^{2}} .
\end{aligned}
$$

[1] E. Schrodinger, Die gegenwärtige situation in der quantenmechanik, Sci. Nat. 23, 807 (1935).

[2] V. Vedral, Quantifying entanglement in macroscopic systems, Nature (London) 453, 1004 (2008).

[3] J. Preskill, Quantum computing in the NISQ era and beyond, Quantum 2, 79 (2018).
[4] R. P. Feynman, in Feynman Lectures on Gravitation, edited by F. Morinigo, W. Wagner, and B. Hatfield (Addison-Wesley, Reading, MA, 1996).

[5] L. Diósi, A universal master equation for the gravitational violation of quantum mechanics, Phys. Lett. A 120, 377 (1987). 
[6] R. Penrose, On gravity's role in quantum state reduction, Gen. Relativ. Gravitation 28, 581 (1996).

[7] S. Bose, A. Mazumdar, G. W. Morley, H. Ulbricht, M. Toroš, M. Paternostro, A. A. Geraci, P. F. Barker, M. S. Kim, and G. Milburn, Spin Entanglement Witness for Quantum Gravity, Phys. Rev. Lett. 119, 240401 (2017).

[8] M. P. Blencowe, Effective Field Theory Approach to Gravitationally Induced Decoherence, Phys. Rev. Lett. 111, 021302 (2013).

[9] I. Pikovski, M. Zych, F. Costa, and Č. Brukner, Universal decoherence due to gravitational time dilation, Nat. Phys. 11, 668 (2015).

[10] A. Belenchia, R. M. Wald, F. Giacomini, E. Castro-Ruiz, Č. Brukner, and M. Aspelmeyer, Information content of the gravitational field of a quantum superposition, Int. J. Mod. Phys. D 28, 1943001 (2019).

[11] D. Carney, P. C. E. Stamp, and J. M. Taylor, Tabletop experiments for quantum gravity: A user's manual, Classical Quantum Gravity 36, 034001 (2019)

[12] M. Aspelmeyer, T. J. Kippenberg, and F. Marquardt, Cavity optomechanics, Rev. Mod. Phys. 86, 1391 (2014).

[13] S. Mancini, V. I. Man'ko, and P. Tombesi, Ponderomotive control of quantum macroscopic coherence, Phys. Rev. A 55, 3042 (1997).

[14] V. Giovannetti, S. Mancini, and P. Tombesi, Radiation pressure induced Einstein-Podolsky-Rosen paradox, Europhys. Lett. 54, 559 (2001).

[15] S. Bose, K. Jacobs, and P. L. Knight, Preparation of nonclassical states in cavities with a moving mirror, Phys. Rev. A 56, 4175 (1997).

[16] S. Mancini, V. Giovannetti, D. Vitali, and P. Tombesi, Entangling Macroscopic Oscillators Exploiting Radiation Pressure, Phys. Rev. Lett. 88, 120401 (2002).

[17] J. Zhang, K. Peng, and S. L. Braunstein, Quantum-state transfer from light to macroscopic oscillators, Phys. Rev. A 68, 013808 (2003).

[18] S. Pirandola, D. Vitali, P. Tombesi, and S. Lloyd, Macroscopic Entanglement by Entanglement Swapping, Phys. Rev. Lett. 97, 150403 (2006).

[19] T. Corbitt, Y. Chen, F. Khalili, D. Ottaway, S. Vyatchanin, S. Whitcomb, and N. Mavalvala, Squeezed-state source using radiation-pressure-induced rigidity, Phys. Rev. A 73, 023801 (2006).

[20] C. Wipf, T. Corbitt, Y. Chen, and N. Mavalvala, Route to ponderomotive entanglement of light via optically trapped mirrors, New J. Phys. 10, 095017 (2008)

[21] X.-Y. Lü, J.-Q. Liao, L. Tian, and F. Nori, Steady-state mechanical squeezing in an optomechanical system via duffing nonlinearity, Phys. Rev. A 91, 013834 (2015).

[22] D. W. C. Brooks, T. Botter, S. Schreppler, T. P. Purdy, N. Brahms, and D. M. Stamper-Kurn, Non-classical light generated by quantum-noise-driven cavity optomechanics, Nature (London) 488, 476 (2012).

[23] T. P. Purdy, P.-L. Yu, R. W. Peterson, N. S. Kampel, and C. A. Regal, Strong Optomechanical Squeezing of Light, Phys. Rev. X 3, 031012 (2013)

[24] N. Aggarwal, T. Cullen, J. Cripe, G. D. Cole, R. Lanza, A. Libson, D. Follman, P. Heu, T. Corbitt, and N. Mavalvala, Room temperature optomechanical squeezing, Nat. Phys. 16, 784 (2020)
[25] J. Chen, M. Rossi, D. Mason, and A. Schliesser, Entanglement of propagating optical modes via a mechanical interface, Nat. Commun. 11, 943 (2020).

[26] K. W. Murch, K. L. Moore, S. Gupta, and D. M. Stamper-Kurn, Observation of quantum-measurement backaction with an ultracold atomic gas, Nat. Phys. 4, 561 (2008).

[27] A. M. Jayich, J. C. Sankey, B. M. Zwickl, C. Yang, J. D. Thompson, S. M. Girvin, A. A. Clerk, F. Marquardt, and J. G. E. Harris, Dispersive optomechanics: A membrane inside a cavity, New J. Phys. 10, 095008 (2008).

[28] A. H. Safavi-Naeini, S. Gröblacher, J. T. Hill, J. Chan, M. Aspelmeyer, and O. Painter, Squeezed light from a silicon micromechanical resonator, Nature (London) 500, 185 (2013).

[29] L. Tian, Robust Photon Entanglement via Quantum Interference in Optomechanical Interfaces, Phys. Rev. Lett. 110, 233602 (2013).

[30] A. A. Rakhubovsky, D. W. Moore, U. Delić, N. Kiesel, M. Aspelmeyer, and R. Filip, Detecting Nonclassical Correlations in Levitated Cavity Optomechanics, Phys. Rev. Appl. 14, 054052 (2020).

[31] S. Barzanjeh, E. S. Redchenko, M. Peruzzo, M. Wulf, D. P. Lewis, G. Arnold, and J. M. Fink, Stationary entangled radiation from micromechanical motion, Nature (London) 570, 480 (2019).

[32] Y.-D. Wang and A. A. Clerk, Reservoir-Engineered Entanglement in Optomechanical Systems, Phys. Rev. Lett. 110, 253601 (2013).

[33] C. Gut, K. Winkler, J. Hoelscher-Obermaier, S. G. Hofer, R. M. Nia, N. Walk, A. Steffens, J. Eisert, W. Wieczorek, J. A. Slater, M. Aspelmeyer, and K. Hammerer, Stationary optomechanical entanglement between a mechanical oscillator and its measurement apparatus, Phys. Rev. Res. 2, 033244 (2020).

[34] C. F. Ockeloen-Korppi, E. Damskägg, J.-M. Pirkkalainen, M Asjad, A. A. Clerk, F. Massel, M. J. Woolley, and M. A. Sillanpää, Stabilized entanglement of massive mechanical oscillators, Nature (London) 556, 478 (2018).

[35] T. A. Palomaki, J. D. Teufel, R. W. Simmonds, and K. W. Lehnert, Entangling mechanical motion with microwave fields, Science 342, 710 (2013).

[36] R. Riedinger, S. Hong, R. A. Norte, J. A. Slater, J. Shang, A. G. Krause, V. Anant, M. Aspelmeyer, and S. Gröblacher, Non-classical correlations between single photons and phonons from a mechanical oscillator, Nature (London) 530, 313 (2016).

[37] R. Riedinger, A. Wallucks, I. Marinković, C. Löschnauer, M. Aspelmeyer, S. Hong, and S. Gröblacher, Remote quantum entanglement between two micromechanical oscillators, Nature (London) 556, 473 (2018).

[38] T. Krisnanda, M. Zuppardo, M. Paternostro, and T. Paterek, Revealing Nonclassicality of Inaccessible Objects, Phys. Rev. Lett. 119, 120402 (2017)

[39] Y. Ma, F. Armata, K. E. Khosla, and M. S. Kim, Optical squeezing for an optomechanical system without quantizing the mechanical motion, Phys. Rev. Res. 2, 023208 (2020).

[40] C. Marletto and V. Vedral, Gravitationally Induced Entanglement between Two Massive Particles is Sufficient Evidence of Quantum Effects in Gravity, Phys. Rev. Lett. 119, 240402 (2017).

[41] L.-M. Duan, G. Giedke, J. I. Cirac, and P. Zoller, Inseparability Criterion for Continuous Variable Systems, Phys. Rev. Lett. 84, 2722 (2000). 
[42] D. E. Chang, C. A. Regal, S. B. Papp, D. J. Wilson, J. Ye, O. Painter, H. J. Kimble, and P. Zoller, Cavity opto-mechanics using an optically levitated nanosphere, Proc. Natl. Acad. Sci. USA 107, 1005 (2009).

[43] O. Romero-Isart, M. L. Juan, R. Quidant, and J. I. Cirac, Toward quantum superposition of living organisms, New J. Phys. 12, 033015 (2010).

[44] N. Kiesel, F. Blaser, U. Delić, D. Grass, R. Kaltenbaek, and M. Aspelmeyer, Cavity cooling of an optically levitated submicron particle, Proc. Natl. Acad. Sci. USA 110, 14180 (2013).

[45] L. Neumeier, T. E. Northup, and D. E. Chang, Reaching the optomechanical strong-coupling regime with a single atom in a cavity, Phys. Rev. A 97, 063857 (2018).

[46] F. Brennecke, S. Ritter, T. Donner, and T. Esslinger, Cavity optomechanics with a Bose-Einstein condensate, Science 322, 235 (2008).

[47] M. Takatsuji, Quantum theory of the optical Kerr effect, Phys. Rev. 155, 980 (1967).

[48] E. Lombardi, F. Sciarrino, S. Popescu, and F. De Martini, Teleportation of a Vacuum-One-Photon Qubit, Phys. Rev. Lett. 88, 070402 (2002).

[49] T. Guerreiro, F. Monteiro, A. Martin, J. B. Brask, T. Vértesi, B. Korzh, M. Caloz, F. Bussières, V. B. Verma, A. E. Lita, R. P. Mirin, S. W. Nam, F. Marsilli, M. D. Shaw, N. Gisin, N. Brunner, H. Zbinden, and R. T. Thew, Demonstration of Einstein-Podolsky-Rosen Steering Using Single-Photon Path Entanglement and Displacement-Based Detection, Phys. Rev. Lett. 117, 070404 (2016).

[50] T. Peyronel, O. Firstenberg, Q.-Y. Liang, S. Hofferberth, A. V. Gorshkov, T. Pohl, M. D. Lukin, and V. Vuletić, Quantum nonlinear optics with single photons enabled by strongly interacting atoms, Nature (London) 488, 57 (2012).

[51] M. Hofheinz, H. Wang, M. Ansmann, R. C. Bialczak, E. Lucero, M. Neeley, A. D. O’Connell, D. Sank, J. Wenner, J. M. Martinis, and A. N. Cleland, Synthesizing arbitrary quantum states in a superconducting resonator, Nature (London) 459, 546 (2009).

[52] L. Martinetz, K. Hornberger, J. Millen, M. S. Kim, and B. A. Stickler, Quantum electromechanics with levitated nanoparticles, npj Quantum Inf. 6, 101 (2020).

[53] P. Krantz, M. Kjaergaard, F. Yan, T. P. Orlando, S. Gustavsson, and W. D. Oliver, A quantum engineer's guide to superconducting qubits, Appl. Phys. Rev. 6, 021318 (2019).

[54] H.-P. Breuer and F. Petruccione, The Theory of Open Quantum Systems (Oxford University, Oxford, 2007).

[55] C. Weedbrook, S. Pirandola, R. García-Patrón, N. J. Cerf, T. C. Ralph, J. H. Shapiro, and S. Lloyd, Gaussian quantum information, Rev. Mod. Phys. 84, 621 (2012).
[56] L. Davidovich, Sub-poissonian processes in quantum optics, Rev. Mod. Phys. 68, 127 (1996).

[57] S. Lloyd and S. L. Braunstein, Quantum Computation over Continuous Variables, Phys. Rev. Lett. 82, 1784 (1999).

[58] T. J. Kippenberg, S. M. Spillane, and K. J. Vahala, KerrNonlinearity Optical Parametric Oscillation in an Ultrahigh- $q$ Toroid Microcavity, Phys. Rev. Lett. 93, 083904 (2004).

[59] P. Rabl, Photon Blockade Effect in Optomechanical Systems, Phys. Rev. Lett. 107, 063601 (2011).

[60] N. Meyer, A. de los Rios Sommer, P. Mestres, J. Gieseler, V. Jain, L. Novotny, and R. Quidant, Resolved-Sideband Cooling of a Levitated Nanoparticle in the Presence of Laser Phase Noise, Phys. Rev. Lett. 123, 153601 (2019).

[61] U. Delić, M. Reisenbauer, D. Grass, N. Kiesel, V. Vuletić, and M. Aspelmeyer, Cavity Cooling of a Levitated Nanosphere by Coherent Scattering, Phys. Rev. Lett. 122, 123602 (2019).

[62] U. Delić, M. Reisenbauer, K. Dare, D. Grass, V. Vuletić, N. Kiesel, and M. Aspelmeyer, Cooling of a levitated nanoparticle to the motional quantum ground state, Science 367, 892 (2020).

[63] D. Windey, C. Gonzalez-Ballestero, P. Maurer, L. Novotny, O. Romero-Isart, and R. Reimann, Cavity-Based 3D Cooling of a Levitated Nanoparticle via Coherent Scattering, Phys. Rev. Lett. 122, 123601 (2019).

[64] M. Paternostro, D. Vitali, S. Gigan, M. S. Kim, C. Brukner, J. Eisert, and M. Aspelmeyer, Creating and Probing Multipartite Macroscopic Entanglement with Light, Phys. Rev. Lett. 99, 250401 (2007)

[65] T. Opatrný, D.-G. Welsch, and W. Vogel, Homodyne detection for measuring internal quantum correlations of optical pulses, Phys. Rev. A 55, 1416 (1997).

[66] A. de los Ríos Sommer, N. Meyer, and R. Quidant, Strong optomechanical coupling at room temperature by coherent scattering, arXiv:2005.10201.

[67] H. Rudolph, K. Hornberger, and B. A. Stickler, Entangling levitated nanoparticles by coherent scattering, Phys. Rev. A 101, 011804(R) (2020).

[68] O. Černotík and R. Filip, Strong mechanical squeezing for a levitated particle by coherent scattering, Phys. Rev. Res. 2 , 013052 (2020).

[69] A. K. Chauhan, O. Černotík, and R. Filip, Stationary Gaussian entanglement between levitated nanoparticles, New J. Phys. 22, 123021 (2020).

[70] J. Gieseler, B. Deutsch, R. Quidant, and L. Novotny, Subkelvin Parametric Feedback Cooling of a Laser-Trapped Nanoparticle, Phys. Rev. Lett. 109, 103603 (2012).

[71] G. P. Conangla, F. Ricci, M. T. Cuairan, A. W. Schell, N. Meyer, and R. Quidant, Optimal Feedback Cooling of a Charged Levitated Nanoparticle with Adaptive Control, Phys. Rev. Lett. 122, 223602 (2019). 